\title{
Advances in Spinal Cord Stimulation for Treatment of Chronic Pain
}

\author{
Jae H. Jung • Mark I. Ignatius • Carolyn F. Schulz • \\ Christopher C. Ornelas
}

Published online: 13 March 2013

(c) Springer Science+Business Media New York 2013

\begin{abstract}
Spinal cord stimulation (SCS) continues to gain validation as an effective and safe treatment option for a variety of chronic pain syndromes, including failed back surgery syndrome, complex regional pain syndrome, refractory angina pectoris, and phantom limb pain. Over the past 30 years, SCS has demonstrated successful outcomes in pain reduction, as well as improvement in quality of life and function. Additionally, there are promising developments under way with cervical spine level SCS and non-painrelated indications. Despite the success of SCS thus far, there is still a paucity of randomized control trials, and further clinical research is warranted to better establish SCS's efficacy across its spectrum of applications.
\end{abstract}

Keywords Spinal cord stimulation - Failed back surgery syndrome Complex regional pain syndrome $\cdot$ Angina pectoris

J. H. Jung $(\bowtie) \cdot$ C. C. Ornelas

Department of Orthopedics, Physical Medicine

and Rehabilitation, The UCLA Spine Center, David Geffen

School of Medicine, 1250 16th Street, Suite $3145 \mathrm{H}$,

Santa Monica, CA 90404, USA

e-mail: jjung@mednet.ucla.edu

M. I. Ignatius - C. F. Schulz

UCLA/VA Greater Los Angeles Healthcare System,

1250 16th Street, Suite 3145H, Santa Monica, CA 90404, USA

e-mail: mi9887@ucla.edu

C. F. Schulz

e-mail: cschulz@ucla.edu

C. C. Ornelas

Department of Physical Medicine and Rehabilitation, Veterans Administration, Greater Los Angeles Healthcare System,

1250 16th Street, Suite 3145H, Santa Monica, CA 90404, USA

e-mail: cornelasmd@mednet.ucla.edu

\section{Introduction}

Spinal cord stimulation (SCS) is an exciting and evolving treatment for chronic pain that reinforces its value by achieving effective results in a variety of chronic pain syndromes, and it continues to expand into more clinical applications. SCS dates back to 1967, when Shealy et al. [1] first began treating cancer pain patients with dorsal column stimulators. It has now become an established treatment option among patients with neuropathic pain syndromes such as failed back surgery syndrome (FBSS), complex regional pain syndrome (CRPS), phantom limb pain, and painful peripheral neuropathies. Recent evidence has demonstrated its utility in coronary artery disease as an effective treatment option for refractory angina pectoris [2•]. Furthermore, there have been advances in alternative neuromodulation uses including treatment of bladder dysfunction, as well as restorative neurology for abnormal lower limb motor control due to Parkinson's disease [3-5].

Although the precise mechanism of action for pain relief with SCS is unknown, the overall premise behind neuromodulation stems from the gate control theory of pain of Melzack and Wall [6], which proposes that stimulation of large myelinated A fibers inhibits the nociceptive activity of the small $\mathrm{A}-\delta$ and $\mathrm{C}$ fibers in the dorsal horn, thus inhibiting the pain signal from reaching the brain. SCS is theorized to stimulate these large-diameter A- $\beta$ fibers, and replace the sensation of pain with a more tolerable paresthesia such as tingling. However, this model would not completely explain all the effects seen with neuromodulation, and current research suggests multiple pathways may be affected by SCS which may contribute to pain reduction. Current proposed mechanisms include inhibition of the excitatory neurotransmitters in the central nervous system through activation of $\gamma$ aminobutyric acid (GABA-B) receptors as well as alteration 
of the hyperactivity of the sympathetic nervous system via adenosine receptors [7••], which could account for the effectiveness of SCS in the treatment of angina. Regardless of the lack of concrete evidence for the SCS mechanism of action, Ramasubbu et al. [8] promoted the field of SCS research by proposing in 2013 that a more thorough understanding of the principles of electrical stimulation and dorsal column mapping will greatly improve the accuracy of lead placement and manipulation of electrical parameters, thereby improving the outcomes associated with suboptimal paresthesia coverage.

SCS implantation is usually a two-step process, consisting of a trial period prior to permanent SCS implant. The preimplantation trial can range from 1 to 2 weeks in duration and is routinely performed via a minimally invasive percutaneous technique, which allows confirmation of pain coverage before committing to a permanent SCS implant. A successful trial is typically gauged at a $50 \%$ or more reduction in pain level from the baseline. The patient and physician may then elect to proceed with the permanent implantation of either percutaneous leads or a paddle lead, which is a more invasive surgical procedure and sometimes requires a laminectomy, and an implantable pulse generator.

The evidence for SCS as a successful treatment in chronic pain syndromes is growing, and over the past decade the results have driven its uses in new directions. This trend and clinical expansion may be related to technologic advancements, discoveries in the neurophysiology of pain, or ongoing training experiences for physicians to hone their technical skills. Particularly, recent success has been demonstrated not only in the more recognized indications such as FBSS and CRPS, but also in the less established SCS applications such as treatment of refractory angina pectoris, phantom limb pain, and cervical spine level SCS.

\section{Failed Back Surgery Syndrome}

The most documented and commonest SCS indication is for chronic back and lower extremity pain following spinal surgical intervention, known as "failed back surgery syndrome" (FBSS). The incidence of FBSS ranges from 10 to $40 \%$ in spinal surgery patients [9]. Numerous case studies on SCS for treatment of FBSS and a series of randomized controlled trials (RCTs) have been published over the last 30 years. The RCTs that have been published tend to support its ongoing use in this population. North et al. [10] reported a significant benefit (50\% or more pain relief) and less opioid drug use in patients who underwent SCS compared with patients who underwent reoperation. Kumar et al. [11] compared SCS with conventional medical management and found SCS to be statistically superior in achieving more than $50 \%$ pain relief $(37 \%$ in the SCS group vs $2 \%$ in the conventional medical management group). Kumar et al. [12] also determined in another study that SCS was effective in providing pain relief over both the short term and the long term, with a follow-up period of 97.6 months. In 2011, North et al. [13••] published the protocol for the ongoing EVIDENCE study. This is a multicenter international RCT with a goal of assessing the clinical and cost-effectiveness of SCS with a rechargeable pulse generator versus reoperation during a 36-month follow-up period. The results of this trial are pending at the time of writing. A number of systematic reviews have also recommended a general consensus for SCS use in FBSS patients. In 2009, Frey et al. [14] indicated there is a level II-1 or level II-2 quality of evidence on the basis of the US Preventive Services Task Force recommendations to support SCS for long-term pain relief in FBSS patients. An earlier systematic review by Taylor et al. [15] in 2005 demonstrated significant heterogeneity between studies in the degree of pain relief and found a "moderate" level of evidence on the basis of the Cochrane Collaboration guidelines for systematic reviews to support it for treatment of FBSS. Taylor [16] later found "grade B" evidence on the basis of the Harbour and Miller grading system to support SCS for treatment of FBSS-refractory neuropathic pain.

SCS candidates are screened prior to the trial phase, and in them other conservative measures have typically failed, including physical therapy, medication management, and other interventional spine procedures. Some FBSS patients may be excluded from SCS selection because of technical difficulties and anatomical deformities, such as kyphoscoliosis, which may preclude proper placement of the device. However, one case report included a 72-year-old with kyphoscoliosis and described alleviation of pain, improvement in physical activity, and discontinuation of opioid use following SCS placement [17].

SCS is not without its risks, as up to $43 \%$ of patients with chronic back and leg pain experienced one or more complications following placement [10]. Complications reported to date are not limited to, but include, the need for revision, infection, defective leads or electrodes, generator or extension cable problems, spinal cord injury, allergic dermatitis caused by the generator, epidural and subcutaneous hematomas, and cerebrospinal fluid leaks [9, 10, 15, 18].

SCS appears to have good clinical outcomes in treatment of FBSS on the basis of the reported cases and limited RCTs published to date. Data from the ongoing EVIDENCE trial comparing SCS with reoperation in FBSS patients may further establish SCS's long-term efficacy and future role in chronic pain management.

\section{Complex Regional Pain Syndrome}

CRPS is a regional pain syndrome of unclear pathogenesis that is thought to be sympathetically driven and is typically 
generated by a prior intervention or trauma. It is divided into two types: type 1, previously known as reflex sympathetic dystrophy, and type 2 , or causalgia, with the latter being the result of an identifiable nerve trauma. Spinal cord stimulator placement for CRPS is one of the commonest indications in the USA when other conservative measures such as medications, physical therapy, or sympathetic blocks have not provided substantial relief. In a 2006 systematic review, Taylor [16] found "grade A" evidence on the basis of the Harbour and Miller grading system to support SCS for treatment of CRPS type 1. Kemler et al. [19] performed a RCT of CRPS type 1 patients that compared SCS and physical therapy with physical therapy alone. They found significant improvements in pain relief at the 2-year follow-up in the SCS group, but without significant functional changes. At the 5-year follow-up, the pain alleviation lost its significant effectiveness compared with the control; however, the patients' perceived satisfaction and desire to repeat the trial remained above $95 \%$ in the SCS group [20]. Kumar et al. [21] also found in 2011 that SCS for treatment of CRPS type 1 patients significantly improved pain, quality of life, and functionality compared with the baseline over a course of 88 months. Kumar et el. recommended early implementation of SCS in the disease continuum, whereas previous studies indicated that an SCS trial should be considered when conservative measures are not efficacious within 12-16 weeks [22]. CPRS typically affects a younger adult population, and SCS trials in the pediatric population are limited. Olsson et al. [23] evaluated SCS as a treatment for seven adolescent girls, between the ages of 11 and 14 years, with exceptionally refractory CRPS type 1 . Five patients experienced complete pain relief and two had partial relief within 2-6 weeks. Interestingly, in four patients the SCS use was gradually diminished and eventually removed. Further studies are warranted to determine what subsets of pediatric CRPS patients are appropriate for SCS and to assess its overall efficacy with additional RCTs.

\section{Cervical Spine Level SCS}

Although most of the published literature for SCS evaluates its efficacy in treatment of conditions such as FBSS and chronic lower extremity pain, there are relatively limited data on cervical spine level SCS indications and outcomes. Cervical spine SCS patients have been included in a few large studies, for example, as part of the CRPS spectrum, with relatively good response [12, 24]. Wolter et al. [24] found average pain scores decreased by $50 \%$ over the course of 6 years. In a study by Forouzanfar et al. [25], similar reductions in pain intensity and improvements in quality of life were found between cervical and thoracolumbar SCS patients. There is concern in the medical community that cervical level stimulation may lead to more complications because of the narrow posterior epidural space and relative mobility of the cervical spine. However, studies thus far have not shown statistical differences in complications compared with thoracolumbar stimulation for treatment of lower extremity pain [24, 25]. Cervical spine level SCS should be considered for treatment of chronic upper limb pain related to failed cervical spine surgical procedures, plexopathies, or nerve root avulsions which are nonresponsive to conservative measures. Recent literature has also shown that cervical spine level SCS may be appropriate for a number of alternative indications, including Raynaud's syndrome and systemic scleroderma [24, 26], chronic cluster headaches [27], and occipital and postherpetic neuralgia [28, 29].

\section{Refractory Angina Pectoris}

Over the past two decades, SCS has come into favor as a reliable and safe nonpharmacologic treatment option in refractory angina pectoris. It was first used to treat refractory angina pectoris in 1987 by Murphy and Giles [30], which resulted in a reduction of angina symptoms as well as decreased use of nitrate medications. Since then, SCS has continued to show promising outcomes in the perceived quality of life of patients, with reductions in hospital admissions and improvements in New York Heart Association functional class [31, 32]. Despite the analgesic effect on angina, neuromodulation does not conceal the symptoms of a myocardial infarction [33].

The ESBY (electrical stimulation versus coronary artery bypass surgery in severe angina pectoris) study by Mannheimer et al. [34] compared the effects of SCS with those of coronary artery bypass graft (CABG) surgery with multiple outcome variables. Both the SCS group and the CABG group showed significant reduction in symptoms and use of nitrate medications, along with improvement in quality of life. Although the CABG group demonstrated greater benefit in exercise capacity, the mortality rate in the early follow-up period was significantly greater, and more cerebrovascular events were observed. Chua and Keogh [35] performed a case series audit of 11 patients with refractory angina pectoris treated with SCS over a 1-year period, and reported significant functional improvements (i.e., 6-min walk distance, exercise duration) as well as symptomatic benefit with decrease in angina attacks. Andréll et al. [36] performed prospective multicenter studies in 2010 to assess the longterm effects of SCS on angina and quality of life in 121 patients with refractory angina pectoris. These patients were followed for approximately 1 year and reported reduction in angina symptoms, decreased use of nitrates, better quality of 
life, and improved Canadian Cardiovascular Society class. Also in 2010, Lanza et al. [2] performed an RCT of 25 patients with refractory angina pectoris who underwent SCS implantation, comparing paresthetic SCS versus subliminal SCS and sham SCS. They concluded that the classic paresthetic SCS was superior to sham SCS in improving angina symptoms and quality of life, but data regarding the efficacy of subliminal SCS were inconclusive. SCS has continued to demonstrate overall safety and effectiveness in the treatment of refractory angina pectoris and should be considered in the appropriate patient population.

\section{Phantom Limb Pain}

Phantom limb pain is another variant of neuropathic pain, with phantom sensations reported in up to $80 \%$ of amputees, with the exact percentage being controversial between studies [9]. In some cases, the pain does not affect the patient's quality of life; however, in others it is excruciatingly debilitating and nonresponsive to conservative measures, including medication, psychotherapy, modalities, and surgical interventions. Although phantom limb pain is well documented, there are limited data on the use of SCS to treat this condition. Initial trials date back to the 1970s, and more recently, amputees with intractable phantom limb pain have undergone SCS trials with mixed results. Viswanathan et al. [18] reported more than $80 \%$ pain relief on a numerical pain scale in all four patients. Katayama et al. [37] noted that $32 \%$ of patients (six of 19) reported more than $80 \%$ long-term pain control with SCS. Patients in whom SCS failed to relive symptoms were evaluated for deep brain or motor cortex stimulation, with no remarkable benefit in controlling phantom limb pain over SCS being reported. Larger RCTs are warranted to make further determinations on its longterm effectiveness in this population.

\section{Conclusion}

Since its inception four decades ago, SCS has emerged as an effective and safe treatment option for a multitude of chronic pain syndromes. SCS may be an initially expensive alternative to conventional medical management; however, studies have shown that it can play a significant role in quality of life improvement and pain relief [15, 38, 39•]. Cost-effectiveness studies to date indicate that the initial SCS health care acquisition costs are greater compared with those of ongoing chronic pain management, however, in the long term follow-up, SCS is more efficacious in pain relief and less costly overall [14, 40]. With the evolving US health care system, the cost-effectiveness and subsequent reimbursement for SCS will depend on the outcomes of future long-term RCTs.

The effectiveness of SCS may improve with advancements in technology, modifications to both percutaneous and paddle lead designs to contain higher contact per paddle ratios (larger pain area coverage), and improved battery life and decreased charging time for the implantable pulse generator. Outcomes may also improve with ongoing scientific research directed at understanding the complete mechanism of action of SCS, as well as a better understanding of the neuromodulation effects on the spinal cord itself. Education of physicians is key in identifying and providing early intervention in the appropriate SCS candidate. Case reports are numerous, and there are innovative pain applications for use of SCS such as treatment of chronic pain from Lyme disease [41, 42] and chronic knee pain following total joint arthroplasty [43]. Despite the growing number of SCS clinical trials, there still remains a need for larger RCTs in both chronic-pain-related and nonpain-related applications.

Disclosure J.H. Jung declares no conflict of interest; M.I. Ignatius declares no conflict of interest; C.F. Schulz declares no conflict of interest; and C.C. Ornelas declares no conflict of interest.

\section{References}

Papers of particular interest, published recently, have been highlighted as:

- Of importance

- Of major importance

1. Shealy CN, Mortimer JT, Reswick JB. Electrical inhibition of pain by stimulation of the dorsal columns: preliminary clinical report. Anesth Analg. 1967;46:489-91.

2. - Lanza GA, Grimaldi R, Greco S, et al. Spinal cord stimulation for the treatment of refractory angina pectoris: a multicenter randomized single-blind study (the SCS-ITA trial). Pain. 2011;152(1):45-52. This is one of the only studies to use sham SCS as a control.

3. Burks FN, Bui DT, Peters KM. Neuromodulation and the neurogenic bladder. Urol Clin North Am. 2010;37(4):559-65.

4. Minassian K, Hofstoetter U, Tansey K, et al. Neuromodulation of lower limb motor control in restorative neurology. Clin Neurol Neurosurg. 2012;114(5):489-97.

5. Agari T, Date I. Spinal cord stimulation for the treatment of abnormal posture and gait disorder in patients with Parkinson's disease. Neurol Med Chir. 2012;52(7):470-4.

6. Melzack R, Wall PD. Pain mechanisms: a new theory. Science. 1965;150(3699):971-9.

7. • Ramasubbu C, Flagg A II, Williams K. Principles of electrical stimulation and dorsal column mapping as it relates to spinal cord stimulation: an overview. Curr Pain Headache Rep. 2013;17(2): 315. This addresses the importance of thorough understanding of neuromodulation principles. 
8. Jeon YH. Spinal cord stimulation in pain management: a review. Korean J Pain. 2012;25(3):143-50.

9. Yampolsky C, Hem S, Bendersky D. Dorsal column stimulator applications. Surg Neurol Int. 2012;3(Suppl 4):S275-89.

10. North RB, Kidd DH, Farrokhi F, et al. Spinal cord stimulation versus repeated lumbosacral spine surgery for chronic pain: a randomized, controlled trial. Neurosurgery. 2005;56(1):98-106; discussion 106-7.

11. Kumar K, Taylor RS, Jacques L, et al. Spinal cord stimulation versus conventional medical management for neuropathic pain: a multicentre randomised controlled trial in patients with failed back surgery syndrome. Pain. 2007;132(1-2):179-88.

12. Kumar K, Hunter G, Demeria D. Spinal cord stimulation in treatment of chronic benign pain: challenges in treatment planning and present status, a 22-year experience. Neurosurgery. 2006;58(3):481-96.

13. •• North RB, Kumar K, Wallace MS, et al. Spinal cord stimulation versus re-operation in patients with failed back surgery syndrome: an international multicenter randomized controlled trial (EVIDENCE study). Neuromodulation. 2011;14(4):330-5; discussion 335-6. This is the first multicenter, multinational RCT to look at the efficacy and cost-effectiveness of SCS in FBSS versus reoperation-ongoing study.

14. Frey ME, Manchikanti L, Benyamin RM, et al. Spinal cord stimulation for patients with failed back surgery syndrome: a systematic review. Pain Physician. 2009;12(2):379-97.

15. Taylor RS, Van Buyten JP, Buchser E. Spinal cord stimulation for chronic back and leg pain and failed back surgery syndrome: a systematic review and analysis of prognostic factors. Spine (Phila Pa 1976). 2005;30(1):152-60.

16. Taylor RS. Spinal cord stimulation in complex regional pain syndrome and refractory neuropathic back and leg pain/failed back surgery syndrome: results of a systematic review and metaanalysis. J Pain Symptom Manage. 2006;31(4 Suppl):S13-9.

17. Atallah J, Armah FA, Wong D, et al. Use of spinal cord stimulator for treatment of lumbar radiculopathy in a patient with severe kyphoscoliosis. Pain Physician. 2008;11(4):555-9.

18. Viswanathan A, Phan PC, Burton AW. Use of spinal cord stimulation in the treatment of phantom limb pain: case series and review of the literature. Pain Pract. 2010;10(5):479-84.

19. Kemler MA, De Vet HC, Barendse GA, et al. The effect of spinal cord stimulation in patients with chronic reflex sympathetic dystrophy: two years' follow-up of the randomized controlled trial. Ann Neurol. 2004;55(1):13-8.

20. Kemler MA, de Vet HC, Barendse GA, et al. Effect of spinal cord stimulation for chronic complex regional pain syndrome type I: five-year final follow-up of patients in a randomized controlled trial. J Neurosurg. 2008;108(2):292-8.

21. Kumar K, Rizvi S, Bnurs SB. Spinal cord stimulation is effective in management of complex regional pain syndrome I: fact or fiction. Neurosurgery. 2011;69(3):566-78; discussion 578-80.

22. Stanton-Hicks M. Complex regional pain syndrome: manifestations and the role of neurostimulation in its management. J Pain Symptom Manage. 2006;31(4 Suppl):S20-4.

23. Olsson GL, Meyerson BA, Linderoth B. Spinal cord stimulation in adolescents with complex regional pain syndrome type I (CRPS-I). Eur J Pain. 2008;12(1):53-9.

24. Wolter T, Kieselbach K. Cervical spinal cord stimulation: an analysis of 23 patients with long-term follow-up. Pain Physician. 2012;15(3):203-12.

25. Forouzanfar T, Kemler MA, Weber WE, et al. Spinal cord stimulation in complex regional pain syndrome: cervical and lumbar devices are comparably effective. Br J Anaesth. 2004; 92(3):348-53.

26. Issa MA, Kim CH. Cervical spinal cord stimulation with 5-column paddle lead in Raynaud's disease. Pain Physician. 2012;15(4):303-9.

27. Wolter T, Kiemen A, Kaube H. High cervical spinal cord stimulation for chronic cluster headache. Cephalalgia. 2011;31(11): $1170-80$

28. Tomycz ND, Deibert CP, Moossy JJ. Cervicomedullary junction spinal cord stimulation for head and facial pain. Headache. 2011; 51(3):418-25.

29. Harke H, Gretenkort P, Ladleif HU, et al. Spinal cord stimulation in postherpetic neuralgia and in acute herpes zoster pain. Anesth Analg. 2002;94(3):694-700.

30. Murphy DF, Giles KE. Dorsal column stimulation for pain relief from intractable angina pectoris. Pain. 1987;28(3):365-8.

31. Gowda RM, Khan IA, Punukollu G, et al. Treatment of refractory angina pectoris. Int J Cardiol. 2005;101(1):1-7.

32. Murray S, Carson KG, Ewings PD, Collins PD, James MA. Spinal cord stimulation significantly decreases the need for acute hospital admission for chest pain in patients with refractory angina pectoris. Heart. 1999;82(1):89-92.

33. Andersen C, Hole P, Oxhøj H. Does pain relief with spinal cord stimulation for angina conceal myocardial infarction? Br Heart J. 1994;71(5):419-21.

34. Mannheimer C, Eliasson T, Augustinsson LE, et al. Electrical stimulation versus coronary artery bypass surgery in severe angina pectoris: the ESBY study. Circulation. 1998;97(12):1157-63.

35. Chua R, Keogh A. Spinal cord stimulation significantly improves refractory angina pectoris-a local experience spinal cord stimulation in refractory angina. Heart Lung Circ. 2005;14(1):3-7.

36. Andréll $\mathrm{P}, \mathrm{Yu} \mathrm{W}$, Gersbach $\mathrm{P}$, et al. Long-term effects of spinal cord stimulation on angina symptoms and quality of life in patients with refractory angina pectoris - results from the European Angina Registry Link Study (EARL). Heart. 2010;96(14): $1132-6$.

37. Katayama Y, Yamamoto T, Kobayashi K, et al. Motor cortex stimulation for phantom limb pain: comprehensive therapy with spinal cord and thalamic stimulation. Stereotact Funct Neurosurg. 2001;77(1-4):159-62.

38. Manca A, Kumar K, Taylor RS, et al. Quality of life, resource consumption and costs of spinal cord stimulation versus conventional medical management in neuropathic pain patients with failed back surgery syndrome (PROCESS trial). Eur J Pain. 2008;12(8):1047-58.

39. - Mekhail NA, Mathews M, Nageeb F, et al. Retrospective review of 707 cases of spinal cord stimulation: indications and complications. Pain Pract. 2011;11(2):148-53. This discusses a large number of indications and complications in SCS patients.

40. Bala MM, Riemsma RP, Nixon J, et al. Systematic review of the (cost)effectiveness of spinal cord stimulation for people with failed back surgery syndrome. Clin J Pain. 2008;24(9):741-56.

41. Shui Y, Tao W, Huang D, et al. Spinal cord stimulation for chronic pain originating from Lyme disease. Pain Physician. 2012; 15(6):511-4.

42. Mearini M, Podetta S, Catenacci E, et al. Spinal cord stimulation for the treatment of upper and lower extremity neuropathic pain due to Lyme disease. Neuromodulation. 2007;10(2):142-7.

43. Lowry AM, Simopoulos TT. Spinal cord stimulation for the treatment of chronic knee pain following total knee replacement. Pain Physician. 2010;13(3):251-6. 